\title{
High speed multi-frequency impedance analysis of single particles in a microfluidic cytometer using maximum length sequences $\dagger$
}

\author{
Tao Sun, David Holmes, Shady Gawad, $\$$ Nicolas G. Green and Hywel Morgan*
}

Received 8th March 2007, Accepted 16th May 2007

First published as an Advance Article on the web 8th June 2007

DOI: $10.1039 / b 703546 b$

A novel impedance spectroscopy technique has been developed for high speed single biological particle analysis. A microfluidic cytometer is used to measure the impedance of single micrometre sized latex particles at high speed across a range of frequencies. The setup uses a technique based on maximum length sequence (MLS) analysis, where the time-dependent response of the system is measured in the time domain and transformed into the impulse response using fast M-sequence transform (FMT). Finally fast Fourier transform (FFT) is applied to the impulse response to give the transfer-function of the system in the frequency domain. It is demonstrated that the MLS technique can give multi-frequency (broad-band) measurement in a short time period (ms). The impedance spectra of polystyrene micro-beads are measured at 512 evenly distributed frequencies over a range from $976.5625 \mathrm{~Hz}$ to $500 \mathrm{kHz}$. The spectral information for each bead is obtained in approximately $1 \mathrm{~ms}$. Good agreement is shown between the MLS data and both circuit simulations and conventional AC single frequency measurements.

\section{Introduction}

In recent years, a large number of microfluidic devices have been developed for characterisation, detection, manipulation and separation of biological particles using electrical, ${ }^{1-3}$ optical, $^{4,5}$ magnetic $^{6}$ and ultrasonic ${ }^{7}$ methods. One area of particular interest is the use of electrical impedance spectroscopy to measure the dielectric properties of single biological particles in microfluidic systems. ${ }^{1-3,8,9}$ Therefore, microimpedance cytometry using micro-fluidic devices could potentially offer a novel high speed method for fast and label-free identification and analysis of cells and micro-sized particles.

Impedance spectroscopy is performed by applying a constant amplitude AC voltage to an unknown system. The impedance is determined by measuring the current flowing through the system using electronic circuitry. In microimpedance cytometry (Fig. 1(a)), the impedance of single particles is measured at high speed as they pass between microfabricated electrodes. ${ }^{1-3,9,10}$ Each pair of top and bottom electrodes defines a measurement volume across the channel. Two pairs of electrodes are positioned close together allowing a differential measurement of the particle to be made. The excitation signal (fixed frequency AC voltage) is applied simultaneously to the two upper microelectrodes. The differential signal from the two lower electrodes is obtained using custom-made detection circuitry. The electronic circuit first measures the particle passing through the first electrode pair, followed immediately by a second measurement as the particle passes through the second pair of electrodes.

Nanoscale Systems Integration Group, School of Electronics and Computer Science, University of Southampton, United Kingdom SO171BJ.E-mail:ts04r@ecs.soton.ac.uk;.hm@ecs.soton.ac.uk

$\dagger$ Electronic supplementary information (ESI) available: Experimental method and supplementary Fig. 1. See DOI: 10.1039/b703546b

* Present address: LMIS4-STI-EPFL, Swiss Federal Institute of Technology, 1015 Lausanne, Switzerland.
The impedance signal depends on particle properties such as size and the intrinsic dielectric properties, which for a cell are dominated by the membrane capacitance and cytoplasmic resistance. The differential measurement system also measures particle speed and reduces measurement noise and instabilities from variations in temperature or composition of the fluid. Particles flow through and are dielectrophoretically focused into a stream along the central line of the channel using negative dielectrophoresis (nDEP) before entering the impedance measurement area. Fig. 1(b) shows a layout of the chip, including the DEP focusing area followed by the measurement electrodes. Four shielding electrodes are placed between the DEP and impedance measurement electrodes to screen the potential from the DEP electrodes. The electrodes at the exit of the measurement channel allow sorting of particles, again by dielectrophoresis, into different outlets.

Conventional impedance analysis is performed by sweeping an excitation signal over a range of frequencies. ${ }^{1-3,9,10}$ The disadvantage of this approach is that scanning the frequency can take several seconds, therefore high speed multi-frequency analysis of moving particles is not possible. One solution involves probing the sample with multi-frequencies simultaneously, as reported by Fuller et al. ${ }^{11}$ These authors used discrete mixers, filters and direct digital signal synthesis circuits with mixed digital and analog application-specific integrated circuit (ASIC) to measure eight frequencies simultaneously. However, the system is complicated and requires a large amount of mixed-signal hardware.

In this paper, we describe a novel, fast and efficient technique for performing broad-band impedance spectroscopy of particles in a time window as short as $1 \mathrm{~ms}$. This technique has significant advantages over traditional techniques and provides a new capability for impedance analysis of particles in a microfluidic format, which is called maximum length sequences (MLS) analysis and is based on obtaining the impulse response of the 


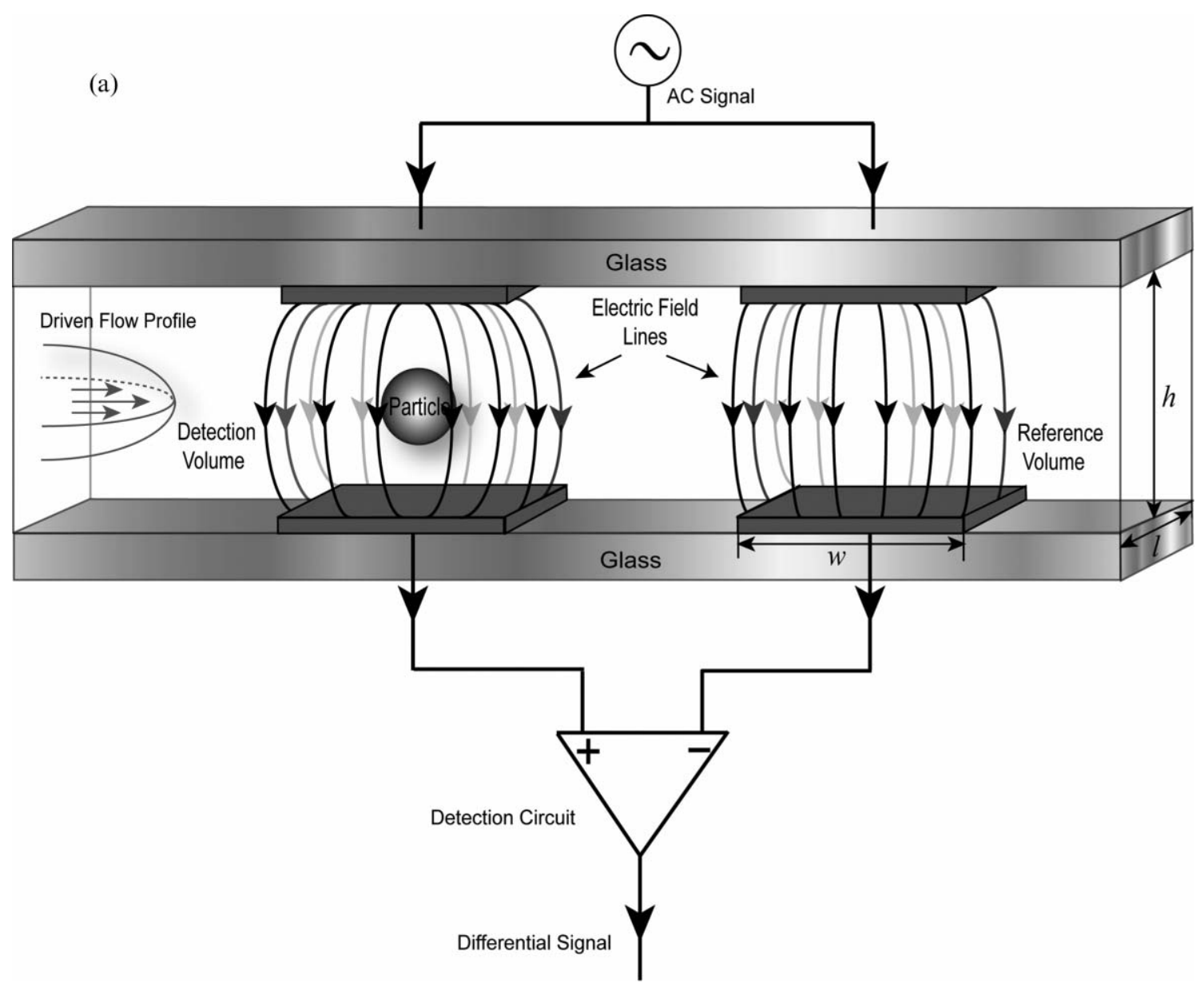

(b)

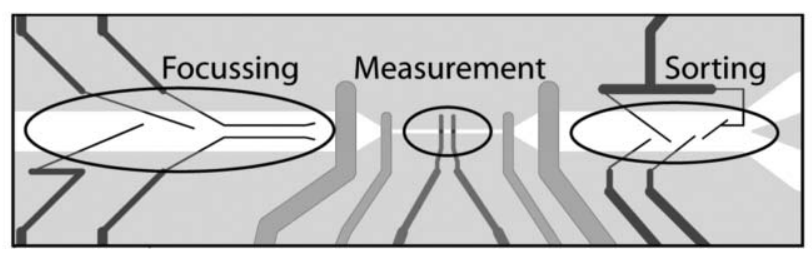

Fig. 1 (a) Diagram showing the impedance cytometer with two pairs of parallel electrodes on the top and bottom of a microfluidic channel. Particles suspended in conducting medium flow, under an external pressure, through the channel and therefore between the electrodes. Also shown is a schematic representation of how the two pairs of electrodes are used to perform a differential measurement of a particle, by taking the difference between the detection volume and the reference volume, $w$ is the width of the electrodes; $l$ the length of the electrodes and $h$ the height of the channel. (b) Diagram showing the layout of the microfluidic chip used for impedance measurement. The focusing and sorting regions of the chip, which work by dielectrophoresis, are shown in the close-up view, as well as the narrower measurement region.

system; the same principle as used in pulse-FFT measurement. ${ }^{12}$ However, the latter method suffers from the disadvantage that the energy of the excitation signal is spread across a wide band of frequencies rather than concentrated at the specific measurement frequency. ${ }^{11}$ Since a short duration pulse cannot deliver enough energy into the system, the signal to noise ratio (SNR) of the pulse-FFT method is worse than the classical discrete frequency system. MLS is a pseudo-random binary sequence (PRBS) and exhibits a uniform power spectral density over a wide frequency band, similar to white noise. In this way, it is a deterministic repeatable signal, and therefore more energy is delivered to the system over the measurement period, compared to the short duration of a pulse in the pulse-FFT technique.
The MLS measurement technique has been used for a number of years in the field of audio engineering and architectural acoustics. ${ }^{13-25}$ However, wider applications of this technique are slowly being recognized. For example, Weckström et al. ${ }^{26}$ used a white-noise-modulated current for the determination of the input impedance of non-spiking neurons. Schneider ${ }^{27}$ proposed using MLS in a multi-frequency EIT-system for observation of long bone fracture healing and Amrani et al. ${ }^{28}$ used MLS as interrogation signals for semi-conducting organic polymer gas sensors. More recently, Rufer et al. ${ }^{29}$ used MLS to determine the mechanical and thermal behavior of MEMS structures. Xiang et al. applied MLS to a laser Doppler vibrometer-based acoustic landmine detection technique ${ }^{30}$ and to sonar systems. ${ }^{31}$ 
To the best of our knowledge, the earliest application of MLS analysis to measure the impulse response of a linear time invariant (LTI) system was reported in $1966,{ }^{13}$ and the MLS theory is now well-established. ${ }^{18-22}$ We have developed the MLS technique for use in electrical impedance spectroscopy and applied it to the analysis of single biological particles flowing within a microfluidic cytometer, thus providing a new high speed analytical technique for characterising the dielectric properties of single particles. In this paper, we briefly explain the principles and characteristics of the MLS measurement technique, and analyse the system through the use of an equivalent circuit model for a homogeneous solid dielectric particle (i.e. bead) in suspension. Experimentally, we demonstrate that MLS can be used to measure the frequency dependent impedance spectrum for polystyrene beads, and demonstrate the substantial hardware cost savings for an MLS system by comparison to a conventional measurement system.

\section{Theory}

\subsection{Maximum length sequences}

MLS comprises a sequence of ones and zeros and is generated recursively using a series of digital shift registers with feedback coefficients control (Fig. 2(a)). It conforms to the linear recurrence:

$$
a_{n}=\left(\sum_{i=1}^{n} c_{i} a_{i}\right) \bmod (2)
$$

In Fig. 2(a), the output digital signal comes from register $a_{n}$ and the newly generated digital signal goes to register $a_{1}$, which is dependent on the states of all the registers and the feedback coefficients. At the same time, each element in every register shifts to the adjacent register on the right. Operation $(\bmod 2)$ is taken to mean modulus 2 or exclusive-OR operation. The feedback coefficients $c_{i}$ are described by the primitive polynomial:

$$
f(x)=1+\sum_{i=1}^{n} c_{i} x^{i}
$$

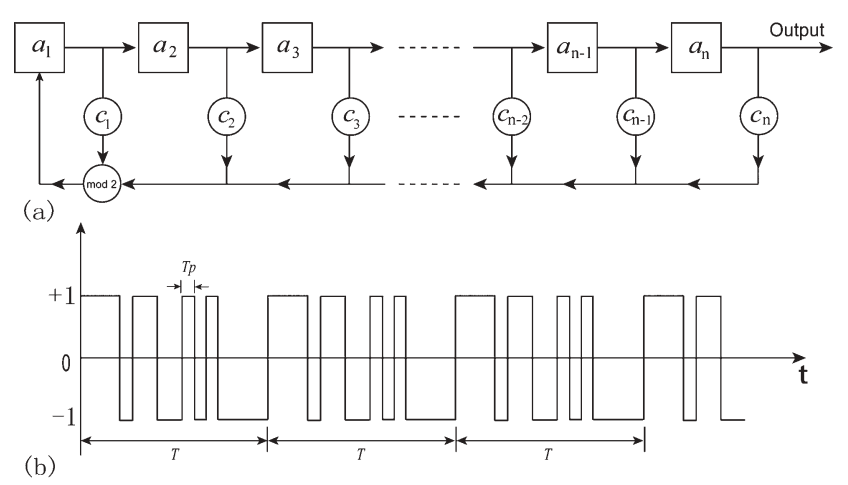

Fig. 2 (a) Diagram showing the structure of the digital shift registers in series with feedback coefficients used to generate the Maximum length sequences (MLS). (b) Diagram showing a typical analog bipolar MLS in the time domain.
With all the shift-registers set to a given state (except all zeros), the $n$-stage registers will generate periodic sequences, with length $L=2^{n}-1$ in one period. Here, $L$ is the length of one MLS with $n$ denoting the order of the sequence and also the number of digital shift registers. This is the so-called maximum length sequence. In practical measurement, the 1 and 0 states are often mapped onto -1 and +1 levels, respectively, to produce a bipolar sequence which is symmetrical about zero. A typical bipolar MLS is shown in Fig. 2(b). The duration of every element in one MLS is represented by $T_{p}$ and the whole period of one sequence is given by $T=L \times T_{p}$.

The MLS analysis technique is based on calculating the cross-correlation function between the input MLS signal and output response of a system. The cross-correlation function is determined by a convolution between the auto-correlation function of the MLS (which is the Dirac delta function with a small DC component) and the impulse response of the system. ${ }^{16}$ Since in practice the MLS system is AC coupled, the DC component can be ignored. This means that the crosscorrelation function of the MLS signal with the output response of the system equals the impulse response of the system. Owing to the unique properties of MLS, the crosscorrelation function can be efficiently calculated using a special algorithm - fast M-sequence transform (FMT), ${ }^{14}$ which is based on the fast Hadamard transform (FHT), consisting of only additions and subtractions. For more details of the theory of MLS, see ref. 18-22.

From signal processing theory, ${ }^{32}$ the impulse response of a system in the time domain is identical to the transfer-function of the system. Therefore, the transfer-function of the entire system can be obtained from the Fourier transform of the impulse response. In terms of single cell analysis, we have recently developed a theoretical model for MLS analysis of particles. ${ }^{33}$ We have shown that with a sufficiently long MLS, and a high sampling rate, the impedance spectrum of a system can be successfully characterized. To improve the computational efficiency of the FFT, we performed a $2^{n}$-point FFT rather than $\left(2^{n}-1\right)$ points. The lowest measured frequency, $f_{\text {min }}$, is determined by the order of the MLS and the sampling rate, $f_{\mathrm{s}}$ (eqn (3a)). This also gives the frequency resolution, which is defined as the difference between two measured adjacent discrete frequencies. From Nyquist sampling theory, ${ }^{32}$ the highest frequency, $f_{\max }$, (eqn (3b)) is half of the sampling rate:

$$
\begin{gathered}
f_{\text {min }}=\frac{f_{\mathrm{s}}}{2^{n}} \\
f_{\text {max }}=\frac{f_{\mathrm{s}}}{2}
\end{gathered}
$$

Therefore, the number of measurement frequencies at one time can be up to $2^{n-1}$ frequencies. The spectral information is collected in one sequence period. For example, if the sampling rate is $1 \mathrm{MHz}$ and the MLS signal is 10th order, a full data set of 512 frequencies can be obtained in approximately $1 \mathrm{~ms}$. Further details of the theoretical analysis can be found in ref. 33 . 


\subsection{Circuit model for a single bead in suspension}

The electrical response of the suspending system can be analyzed using equivalent circuits. The electrical circuit for a single shelled cell in suspension has been reported by several authors. ${ }^{1-3,9,34}$ Here, we derive the equivalent circuit model for a solid particle (bead) in suspension. A system with two parallel facing electrodes (Fig. 1) is represented by the circuit shown in Fig. 3(a). For a dilute (low volume fraction) suspension of particles, the dielectric properties of the system are well described by Maxwell's mixture equation. ${ }^{35}$ The equivalent complex permittivity of the mixture is:

$$
\tilde{\varepsilon}_{\text {mix }}=\tilde{\varepsilon}_{\mathrm{m}} \frac{1+2 \Phi\left(\frac{\tilde{\varepsilon}_{\mathrm{p}}-\tilde{\varepsilon}_{\mathrm{m}}}{\tilde{\varepsilon}_{\mathrm{p}}+2 \tilde{\varepsilon}_{\mathrm{m}}}\right)}{1-\Phi\left(\frac{\tilde{\varepsilon}_{\mathrm{p}}-\tilde{\varepsilon}_{\mathrm{m}}}{\tilde{\varepsilon}_{\mathrm{p}}+2 \tilde{\varepsilon}_{\mathrm{m}}}\right)}
$$

where $\tilde{\varepsilon}_{\text {mix }}$ is the complex permittivity of the mixture and $\Phi$ is the volume fraction (ratio of the particle volume to the detection volume). $\tilde{\varepsilon}_{\mathrm{p}}$ and $\tilde{\varepsilon}_{\mathrm{m}}$ are the complex permittivities of the particle and medium respectively. In general the complex permittivity of a material is given by: $\tilde{\varepsilon}=\varepsilon-j \sigma / \omega$, where $\varepsilon$ is the permittivity, $\sigma$ the conductivity, $j^{2}=-1$ and $\omega$ the angular frequency. The effective conductivity of the bead, $\sigma_{\mathrm{p}}$, is the sum of the bulk conductivity, $\sigma_{\text {pbulk }}$ and the conductivity around the bead due to the surface conductance $K_{\mathrm{s}}:{ }^{36}$

$$
\sigma_{\mathrm{p}}=\sigma_{\mathrm{pbulk}}+2 \frac{K_{\mathrm{s}}}{R}
$$

where $R$ is the radius of the bead. Generally, the bulk

(a)
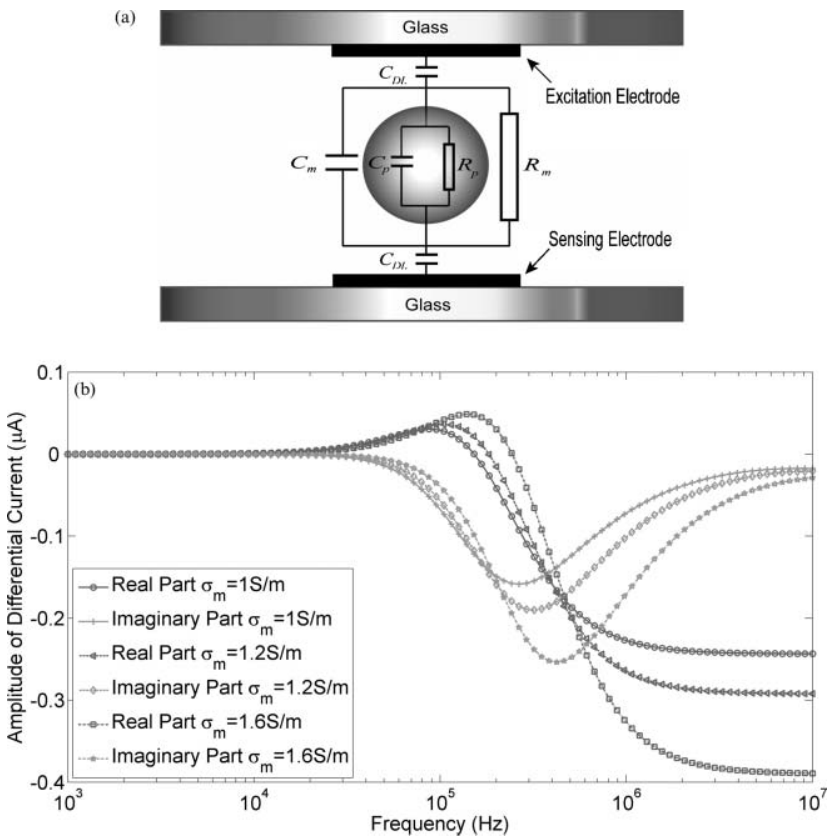

Fig. 3 (a) A representation of the equivalent circuit model for a single homogeneous particle in suspension between the electrodes. $R_{\mathrm{m}}$ and $R_{\mathrm{p}}$ are the resistance of the medium and bead respectively; $C_{\mathrm{m}}$ and $C_{\mathrm{p}}$ are the capacitance of the medium and bead respectively. The electrical double layer (DL) is modeled by the capacitor, $C_{\mathrm{DL}}$. (b) Variation in the real and imaginary part of the differential current signal for three different values of medium conductivity indicated by the legend. conductivity of the bead is negligible and the effective conductivity is determined by the surface conductance and the size of the bead.

To obtain values for the individual electrical components, we applied Laplace and Fourier transforms ${ }^{37}$ to eqn (4) giving:

$$
\tilde{\varepsilon}_{\text {mix }}=\varepsilon_{\infty}+\frac{\sigma_{0}}{j \omega}+\frac{\Delta \varepsilon}{1+j \omega \tau}
$$

with $\varepsilon_{\infty}=\frac{A_{1}}{A_{2}} \varepsilon_{\mathrm{m}} \quad \sigma_{0}=\frac{A_{5}}{A_{2}} B \quad \Delta \varepsilon=\frac{A_{5}}{A_{2}}(1-B) \tau \quad \tau=\frac{A_{2}}{A_{4}}$, where $\varepsilon_{\infty}$ is the limiting high frequency permittivity, $\sigma_{0}$ is the limiting low frequency conductivity, $\Delta \varepsilon$ is the magnitude of the dielectric dispersion, and $\tau$ is the relaxation time constant characterizing the Maxwell-Wagner interfacial polarization at the interface between the bead and suspending medium.

The relevant coefficients are given by:

$A_{1}=\left(\varepsilon_{\mathrm{p}}+2 \varepsilon_{\mathrm{m}}\right)+2 \Phi\left(\varepsilon_{\mathrm{p}}-\varepsilon_{\mathrm{m}}\right) \quad A_{2}=\left(\varepsilon_{\mathrm{p}}+2 \varepsilon_{\mathrm{m}}\right)-\Phi\left(\varepsilon_{\mathrm{p}}-\varepsilon_{\mathrm{m}}\right)$

$A_{3}=\left(\sigma_{\mathrm{p}}+2 \sigma_{\mathrm{m}}\right)+2 \Phi\left(\sigma_{\mathrm{p}}-\sigma_{\mathrm{m}}\right) \quad A_{4}=\left(\sigma_{\mathrm{p}}+2 \sigma_{\mathrm{m}}\right)-\Phi\left(\sigma_{\mathrm{p}}-\sigma_{\mathrm{m}}\right)$

$A_{5}=A_{1} \sigma_{\mathrm{m}}+\left(A_{3}-\frac{A_{1} A_{4}}{A_{2}}\right) \varepsilon_{\mathrm{m}} \quad B=\frac{A_{2} A_{3}}{A_{4} A_{5}} \sigma_{\mathrm{m}}$

According to eqn (6), the values of the electric components in Fig. 3(a) are therefore:

$$
\begin{gathered}
R_{\mathrm{m}}=\frac{1}{\sigma_{0} l \kappa} \\
C_{\mathrm{m}}=\varepsilon_{\infty} l \kappa \\
R_{\mathrm{p}}=\frac{\tau}{C_{\mathrm{p}}} \\
C_{\mathrm{p}}=\Delta \varepsilon l \kappa
\end{gathered}
$$

with $\kappa=\frac{K(k)}{K^{\prime}(k)} k=\tanh \left(\frac{\pi w}{2 h}\right)$ where $\kappa$ is the cell constant, solved using the Schwarz-Christoffel mapping method, to take into account the non-uniform field effect. ${ }^{2,9} K(k)$ is the complete elliptic integral of the first kind, $K^{\prime}(k)$ is the complementary integral and $k$ is the modulus of the elliptic function.

Including the effect of the electrical double layer (DL), the complex impedance of the mixture (bead plus medium) becomes:

$$
\tilde{Z}_{\text {mix_DL }}=\frac{2}{j \omega C_{\mathrm{DL}}}+\frac{R_{\mathrm{m}} R_{\mathrm{p}}}{R_{\mathrm{m}}+R_{\mathrm{p}}+j \omega R_{\mathrm{m}} R_{\mathrm{p}}\left(C_{\mathrm{m}}+C_{\mathrm{p}}\right)}
$$

and the complex impedance of the system without the bead $(\Phi=0)$ is:

$$
\tilde{Z}_{\text {med_DL }}=\frac{2}{j \omega C_{\mathrm{DL}}}+\frac{R_{\mathrm{m}}}{1+j \omega R_{\mathrm{m}} C_{\mathrm{m}}}
$$

According to eqn (8) and (9), the differential current signal from the electronic circuit, $I_{\text {diff }}$ can be calculated as:

$$
I_{\text {diff }}=\frac{\tilde{Z}_{\text {med_DL }}-\tilde{Z}_{\text {mix_DL }}}{\tilde{Z}_{\text {mix_DL }} \tilde{Z}_{\text {med_DL }}} V
$$

where $V$ is the applied voltage onto the system. 
In a differential measurement scheme, the output voltage is proportional to the differential current. Eqn (10) can then be used to analyze variations in the output response of the system caused by changes in the properties of the bead, medium or chip geometry. Fig. 3(b) shows typical examples of the complex differential current variations for three different values of medium conductivity. Calculations were performed in Matlab $^{\mathrm{TM}}$ (Mathworks Inc., USA) with the following parameters: $\varepsilon_{\mathrm{o}}=8.854 \times 10^{-12} \mathrm{Fm}^{-1}, \varepsilon_{\mathrm{m}}=78 \varepsilon_{\mathrm{o}}, \varepsilon_{\mathrm{p}}=$ $2.55 \varepsilon_{\mathrm{o}}, K_{\mathrm{s}}=1.2 \mathrm{nS}, R=5 \mu \mathrm{m}$ and $C_{\mathrm{DL}}=60 \mathrm{pF}$ with geometric dimensions $w=h=l=20 \mu \mathrm{m}$. Eqn (10) also enables the optimal frequency for maximum sensitivity to be determined as shown by Fig. 3(b).

\section{Experimental}

\subsection{Microfluidic cytometer chip}

The microfluidic chips (shown in Fig. 1) were fabricated using photolithography and full wafer thermal bonding. The microfluidic channel was fabricated from a photosensitive polyimide precursor and had dimensions of $20 \mu \mathrm{m}$ wide by $20 \mu \mathrm{m}$ high in the impedance measurement section. The microelectrodes had width and gap of $20 \mu \mathrm{m}$, were made by photolithography and consisted of a layer of titanium and platinum. Individual chips were released from the wafers by dicing with a diamond saw. Inlet and outlet holes were drilled in individual chips for fluid access. Full details of the chip microfabrication have been reported elsewhere. ${ }^{1,2,38}$

\subsection{Measurement system setup}

In order to evaluate the accuracy of the MLS system, the impedance spectra of single beads were measured using both the MLS and conventional single frequency AC methods.

Details of the AC single frequency measurement system and associated microfluidics can be found in the ESI $\dagger$. The impedance measurement setup has been reported previously. $1,3,8,9$ Two AC excitation signals (one at low frequency, e.g. $0.5 \mathrm{MHz}$ and one at high frequency, typically $2-5 \mathrm{MHz}$ ) are mixed and applied to the cytometer chip. The electrical current passing through the two detection volumes is converted into voltage signals by a trans-impedance amplifier. The two signals are measured and amplified in a differential circuit. Two lock-in amplifiers (SR844 Stanford Research Systems. Inc., USA) demodulate the in-phase and out-ofphase signals at each frequency, whilst rejecting noise at other frequencies. The output signals from the lock-in amplifiers are sampled with a 16 bit data acquisition card (NI6251, National Instruments Inc., USA). Data analysis is performed using software written in MATLAB ${ }^{\mathrm{TM}}$ to extract the impedance information, such as the magnitude and phase.

The MLS measurement system is shown in Fig. 4, where the signal generators and lock-in amplifiers of a conventional system are replaced with the MLS signal, which is generated by custom-designed software and clocked at the same rate as the A/D sampling rate. After conversion of the digital MLS signal to an analogue voltage, the signal is applied to the electrodes. The same electronics measures the differential signal; the

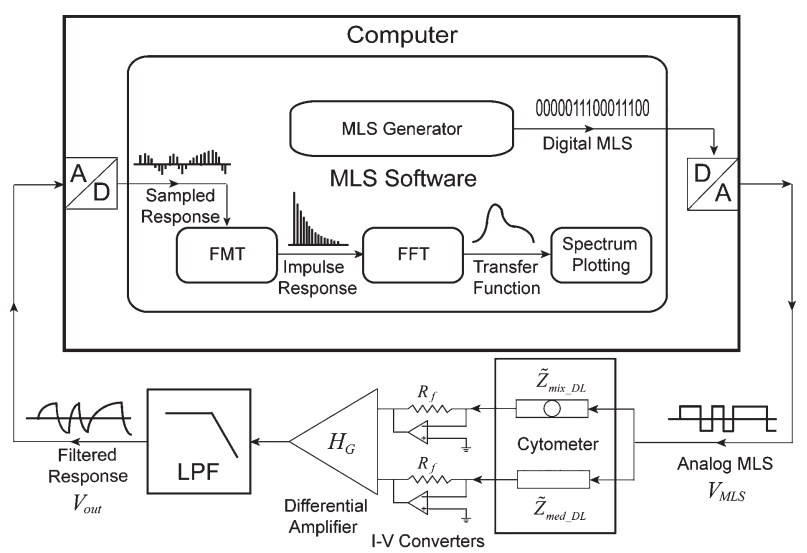

Fig. 4 Schematic diagram showing the MLS impedance measurement system.

output is low pass filtered, then sampled by an A/D converter. The filter, D/A and A/D converters are all integrated into a single data acquisition card (National Instruments NI 6251). Software is used to transform the sampled output response into the impulse response of the system with the fast M-sequence transform (FMT). The transfer-function of the system is obtained from the FFT and finally the impedance information determined.

The transfer-function of the MLS measurement system, $H_{\text {sys }}$, is related to the impedance of the particle in suspension, which can be expressed as:

$$
H_{\text {sys }}=\frac{V_{\text {out }}}{V_{\text {MLS }}}=\frac{\tilde{Z}_{\text {mix_DL }}-\tilde{Z}_{\text {med_DL }}}{\tilde{Z}_{\text {mix_DL }} \tilde{Z}_{\text {med_DL }}} R_{\mathrm{f}} H_{\mathrm{G}} H_{\mathrm{DAQ}}
$$

As shown in Fig. 4, $V_{\text {MLS }}$ is the MLS excitation signal. $V_{\text {out }}$ is the response of the system before the A/D conversion. $R_{\mathrm{f}}$ is the resistance of the feedback resistor in the current-to-voltage (I-V) converter and $H_{\mathrm{G}}$ is the transfer-function of the amplifier. $H_{\mathrm{DAQ}}$ is the transfer-function of the NI 6251 DAQ, which is dominated by the characteristics of the antialiasing low pass filter.

\subsection{Particle preparation and focusing}

Carboxy-polystyrene beads of 5.49 and $7.18 \mu \mathrm{m}$ diameter (Bangs Laboratories Inc., USA) were obtained from Molecular Probes. Prior to use, they were sonicated to separate clumped particles and suspended in phosphate buffered saline (PBS), conductivity $1.6 \mathrm{Sm}^{-1}$.

In order to minimise fluctuations in the position of the particles within the sensing regions, the beads were dielectrophoretically focused prior to passing through the impedance detection area. The voltage used for DEP focusing was $5 \mathrm{Vpp}$ at $8 \mathrm{MHz}$, anti-phase to diagonally opposite electrodes. $^{38,39}$

\section{Results and discussion}

\subsection{Data analysis}

Samples of the two sizes of beads were prepared and mixed in PBS (phosphate buffered saline) at a ratio of approximately 
$10: 1(5.49: 7.18 \mu \mathrm{m})$. Measurements were performed using a 10th order MLS with the sampling rate limited to $1 \mathrm{MHz}$ by the data acquisition card. Therefore, from eqn (3a) and (3b), the lowest frequency is $976.5625 \mathrm{~Hz}$ and the upper frequency limit is $500 \mathrm{kHz}$. The complex transfer-function spectrum of the system is characterized within every discrete time interval. The time interval for each spectrum is the period of an individual sequence, which in this work is approximately $1 \mathrm{~ms}$. Therefore, the MLS technique allows identification of the transfer-function of the system in both time and frequency domain.

Because the particle is moving, the system is not timeinvariant. Therefore, to calculate the impedance of a particle, the time instant at which the particle resides in the detection zone must be established. The impedance data of the beads are extracted from the peak values of the time-varying transferfunction-see Fig. 5(a). This figure shows the real part of the transfer-function over a four second period, for one discrete frequency $(488.28 \mathrm{kHz})$, showing the difference in the signal amplitude for different sizes of beads. Each pair of peaks (one positive and one negative) corresponds to the passage of a single bead through the detection volume. The data plotted in the figure was processed using a Savitzky-Golay $(\mathrm{S}-\mathrm{G})$ finite impulse response smoothing filter, which reduces the background noise. Baseline drift was removed by subtracting the averaged background noise level.

Fig. 5(b) shows a typical impedance signal for two sizes of beads, in this case plotted for four discrete frequencies: $97.656 \mathrm{kHz}, 195.31 \mathrm{kHz}, 390.625 \mathrm{kHz}$ and $488.28 \mathrm{kHz}$. The figure demonstrates how MLS measures the impedance of every bead at many different frequencies at the same time. For 10th order MLS, all 512 individual frequencies are obtained in a single measurement time of approximately $1 \mathrm{~ms}$. Fig. 5(c) shows a histogram, where the number of beads is plotted against the real part of the impedance measured at $488.28 \mathrm{kHz}$. It shows two discrete populations, with distributions in the sizes of the two populations. The spread in the data is partly due to drift in the position and velocity of the particle passing through the detection area as well as an inherent distribution in particle size (coefficient of variance, $\mathrm{CV}=5 \%$ ).

\subsection{Data validation}

In order to validate the impedance spectra of the beads, the MLS measurement results for $5.49 \mu \mathrm{m}$ beads were compared with PSpice circuit simulation (OrCAD Capture CIS, Cadence Design Systems, Inc., USA) and also experimental results obtained using a single frequency AC measurement system. The circuit simulations were performed using the equivalent circuit model with identical dielectric parameters for the medium and a single $5.49 \mu \mathrm{m}$ bead as shown in section 2.2 (Fig. 3(a)). The PSpice models for the active elements (operational amplifiers, etc.) were obtained from the manufacturers. As discussed previously, MLS measures the entire transfer-function of the system, and the characteristic of the low pass filter in the $\mathrm{A} / \mathrm{D}$ card must be considered in the analysis. The transfer-function of the filter was measured by connecting the $\mathrm{D} / \mathrm{A}$ and $\mathrm{A} / \mathrm{D}$ ports together with a coaxial cable. ${ }^{40}$ For AC single frequency measurement, the
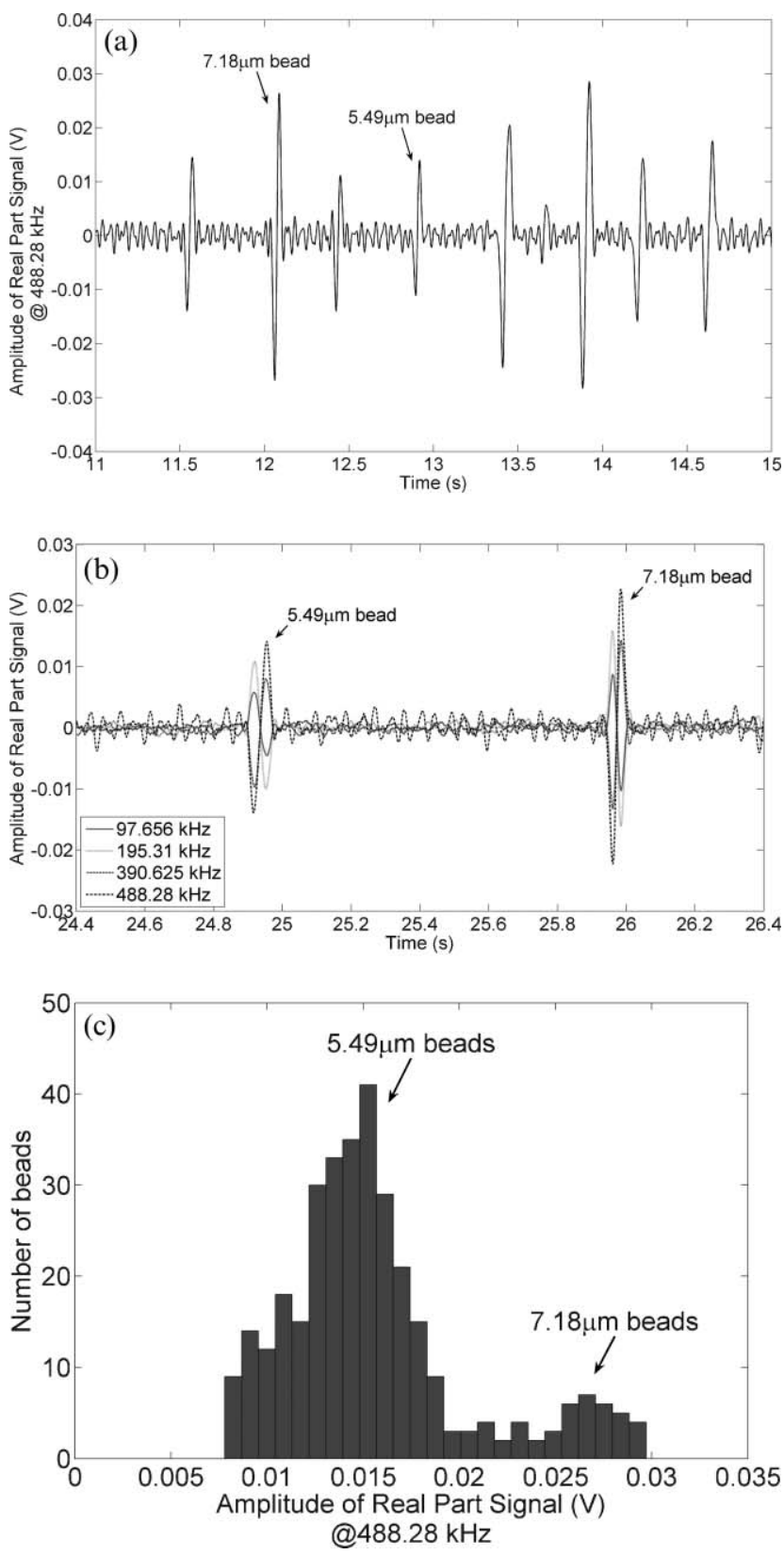

Fig. 5 (a) A captured time window, showing the transfer-function signal, measured at $488.28 \mathrm{kHz}$ for a mixture of beads suspended in PBS passing through the detection area and the difference in the signal amplitude for different sizes of bead. (b) Impedance signals as a function of time, for two different beads, at four selected frequencies: $97.656 \mathrm{kHz}, 195.31 \mathrm{kHz}, 390.625 \mathrm{kHz}$ and $488.28 \mathrm{kHz}$. (c) Histogram of the impedance of the beads measured at $488.28 \mathrm{kHz}$, where the two bead populations can clearly be identified.

demodulated in-phase and out-of-phase signals from the lockin amplifier correspond to the real and imaginary part of the complex impedance signal, respectively. Fig. 6 shows the MLS data, the single frequency data and the PSpice simulation results for $5.49 \mu \mathrm{m}$ beads. The MLS measurement provides 512 discrete frequency points obtained in approximately $1 \mathrm{~ms}$. The AC single frequency measurement data for ten different frequencies was measured over several minutes and is the average signal for 200 beads at every frequency. The figure 


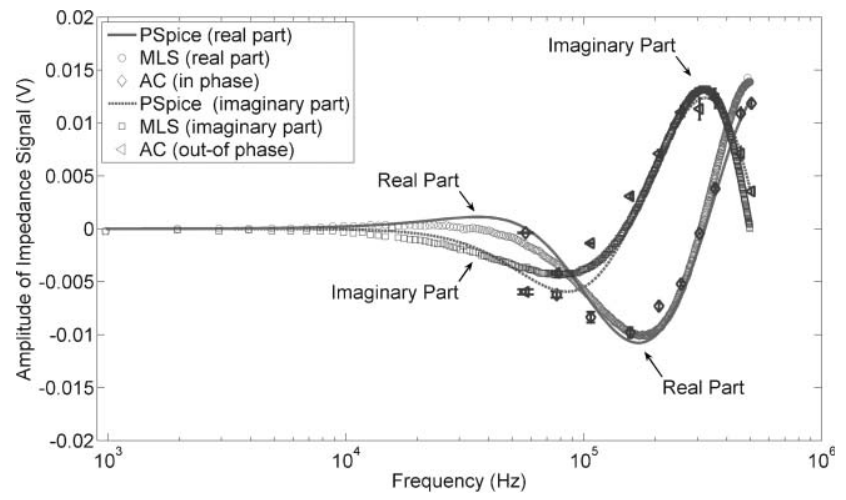

Fig. 6 Real and imaginary part of the impedance spectrum for the $5.49 \mu \mathrm{m}$ bead obtained from PSpice simulation, MLS measurements and $\mathrm{AC}$ single frequency measurements, showing good agreement.

shows good agreement for all three methods, indicating that the MLS system correctly measures the impedance spectrum for a single particle.

\section{Conclusion}

The maximum length sequence measurement technique has been used to characterize the impedance spectrum of single micro-particles flowing through a microfluidic cytometer. The MLS technique measures the impedance across a range of frequencies using a pseudorandom noise as the excitation signal. Compared with standard single frequency (or swept) AC measurements, the hardware costs are substantially less, because no signal generator and lock-in amplifier are required. This simplifies the architecture and enables miniaturization of the entire measurement system. We have theoretically demonstrated the principles of the MLS technique for measuring the impedance spectrum of single particles in suspension. Experimentally, we have shown that the system is able to measure impedance variations from small polystyrene particles and differentiate them by size. The MLS method gives the impedance spectrum of an individual particle in a short time period with high frequency resolution.

The MLS measurement system described in this paper is still in development. The major limitation is the upper frequency limit of $500 \mathrm{kHz}$, which is limited by the sampling rate of the data acquisition card. Within this frequency range, the measured impedance signal can only provide information on the size of particles because the low-frequency sensitivity is limited by the double layer. Much more information on the properties of particles is available if the measurement spectrum could be extended to higher frequencies.

\section{Acknowledgements}

The work is partly supported by the funding from Life Science Initiative, University of Southampton. Dr Gawad would like to acknowledge the funding from the SNSF fellowship.

\section{References}

1 S. Gawad, L. Schild and Ph. Renaud, Lab Chip, 2001, 1, 76-82.

2 S. Gawad, K. Cheung, U. Seger, A. Bertsch and Ph. Renaud, Lab Chip, 2004, 4, 241-251.

3 K. Cheung, S. Gawad and Ph. Renaud, Cytometry Part A, 2005, 65A, 124-132.

4 B. Yao, G. A. Luo, X. Feng, W. Wang, L. X. Chen and Y. M. Wang, Lab Chip, 2004, 4, 603-607.

5 D. Holmes, H. Morgan and N. G. Green, Biosens. Bioelectron., 2006, 21, 1621-1630.

6 N. Pamme, Lab Chip, 2006, 6, 24-38.

7 M. Wiklund and H. M. Hertz, Lab Chip, 2006, 6, 1279-1292.

8 H. Morgan, D. Holmes and N. G. Green, Curr. Appl. Phys., 2006, 6, 367-370.

9 H. Morgan, T. Sun, D. Holmes, S. Gawad and N. G. Green, J. Phys. D: Appl. Phys., 2007, 40, 61-70.

10 S. Gawad, Ph.D thesis, Lausanne EPFL, Switzerland, 2004.

11 C. K. Fuller, J. Hamilton, H. Ackler, P. Krulevitch, B. Boser, A. Eldredge, F. Becker, J. Yang and P. Gascoyne, Proc. $\mu T A S$ 2000, 2000, 265-268

12 Y. Liu, W. Abel, J. Janette, F. Belch, R. J. Tweedie, US Pat., $5691633,1997$.

13 W. D. T. Davies, Control, 1966, 302-304.

14 M. Cohn and A. Lempel, IEEE. Trans. Inf. Theory, 1977, 135-137.

15 M. R. Schroeder, J. Acoust. Soc. Am., 1979, 66, 497-500.

16 J. Borish and J. B. Angell, J. Audio Eng. Soc., 1983, 31, 478-488.

17 Y. Ando, Concert Hall Acoustics, Springer, New York, 1985, pp. 103-109.

18 J. Borish, JAES, 1985, 33, 888-891.

19 D. D. Rife and J. Vanderkooy, J. Audio Eng. Soc., 1989, 37, 419-444.

20 N. Xiang, Signal Process., 1992, 28, 139-152.

21 J. Vanderkooy, J. Audio Eng. Soc., 1994, 42, 219-231.

22 M. Vorlander and M. Kob, Appl. Acoust., 1997, 52, 239-258.

23 D. G. Ciric, TELSIKS'99, 1999, 653-656.

24 N. Xiang and M. R. Schroeder, J. Acoust. Soc. Am., 2003, 113, $2754-2761$.

25 N. Xiang, J. N. Daigle and M. Kleiner, J. Acoust. Soc. Am., 2005, 117, 1889-1894.

26 M. Weckström, E. Kouvalainen and M. Juusola, Pfügers Arch., 1992, 421, 469-472.

27 I. Schneider, Proc. 18th Annu. Int. Conf. IEEE EMBS, 1996, 1934-1935.

28 M. E. H. Amrani, R. M. Dowdeswell, P. A. Payne and K. C. Persaud, Sens. Actuators, B, 1998, 47, 118-124.

29 L. Rufer, S. Mir, E. Simeu and C. Domingues, Des. Test Integr. Packag. of MEMS/MOEMS, 2003, 50-55.

30 N. Xiang and J. M. Sabatier, IEEE Geosci. Remote Sens. Lett., 2004, 1, 292-294.

31 N. Xiang and D. Z. Chu, Proc. ICSP, 2004, 2433-2436.

32 A. V. Oppenheim, A. S. Willsky and I. T. Young, Signal and Systems, Prentice-Hall International, Inc., London, 1983.

33 T. Sun, S. Gawad, N. G. Green and H. Morgan, Meas. Sci. Technol., submitted.

34 K. R. Foster and H. P. Schwan, Crit. Rev. Biomed. Eng., 1989, 17, 25-104.

35 J. C. Maxwell, A Treatise on Electricity and Magnetism, Dover Press, New York, 1954.

36 M. P. Hughes and H. Morgan, Anal. Chem., 1999, 71, 3441-3445.

37 T. Sun, S. Gawad, N. G. Green and H. Morgan, J. Phys. D: Appl. Phys., 2007, 40, 1-8.

38 D. Holmes, J. K. She, P. L. Roach and H. Morgan, Lab Chip, 2007, DOI: $10.1039 / \mathrm{b} 707507 \mathrm{n}$.

39 D. Holmes, M. E. Sandison, N. G. Green and H. Morgan, IEE Proc. Nanobiotechnol., 2005, 152, 129-135.

40 S. Gawad, T. Sun, N. G. Green and H. Morgan, Rev. Sci. Instrum., 2007, 78, DOI: $10.1063 / 1.2737751$. 